\title{
Tuberculosis incidence in the Irish Traveller population in Ireland from 2002 to 2013
}

\author{
R. F. O'TOOLE ${ }^{1,2} *$, S. JACKSON ${ }^{3}$, A. HANWAY 4 , J. O'DONNELL ${ }^{3}$, \\ C. M. COMISKEY ${ }^{4}$, T. R. ROGERS ${ }^{2}$ AND D. O'FLANAGAN ${ }^{3}$ \\ ${ }^{1}$ Breathe Well NHMRC Centre of Research Excellence, School of Medicine, University of Tasmania, Hobart, \\ Australia \\ ${ }^{2}$ Department of Clinical Microbiology, School of Medicine, Trinity College Dublin, Ireland \\ ${ }^{3}$ Health Protection Surveillance Centre, Dublin, Ireland \\ ${ }^{4}$ School of Nursing and Midwifery, Trinity College Dublin, Ireland
}

Received 16 October 2014; Final revision 6 January 2015; Accepted 18 January 2015

\section{SUMMARY}

The health status of the Irish Traveller ethnic minority is low compared to the general population in Ireland in terms of infant mortality rates and life expectancies. Respiratory disease is an area of health disparity manifested as excess mortalities in Traveller males and females. In this study, we examined the available data with regard to tuberculosis (TB) notifications in Ireland from 2002 to 2013. We found an increase in TB notifications in Irish Travellers from 2010 onwards. This resulted in a crude incidence rate for TB in Irish Travellers that was about threefold higher than that of the white Irish-born population in 2011 and 2012. An outbreak of TB in Irish Travellers in 2013 increased this differential further, but when outbreak-linked cases were excluded, a higher incidence rate was still observed in Irish Travellers relative to the general population and to white Irish-born. The mean age of a TB patient was 26 years in Irish Travellers compared to 43 years in the general population, and 49 years in white Irish-born. Based on available data, Irish Travellers exhibit a higher incidence rate and younger age distribution of TB compared to white Irish-born and the general population. These observations emphasize the importance of routine use of ethnicity identifiers in the management of TB and other notifiable communicable illnesses in Ireland. They also have implications for the orientation of preventive services to address health disparities in Irish Travellers and other ethnic minority groups.

Key words: Epidemiology, mycobacteria, tuberculosis (TB).

\section{INTRODUCTION}

A survey of the Irish Traveller population in Ireland and Northern Ireland published in 2010 found that

\footnotetext{
* Author for correspondence: Dr R.F. O’Toole, Breathe Well NHMRC Centre of Research Excellence, School of Medicine,
}

infant mortality rates are about 3.5 times higher than in the general population [1]. Life expectancy at birth in Irish Traveller males and females is reportedly $15 \cdot 1$ and $11 \cdot 5$ years lower, respectively, compared to the national average [1]. In the 2011 Census of England and Wales, the median age of the Gypsy or Irish Travellers group was 26 years compared to the national median age of 39 years in the in the UK [2]. A local baseline census study indicated poor life 
expectancy for Gypsies and Travellers in Leeds compared to the general Leeds population [3]. Currently, our understanding of the burden of communicable illnesses in the Traveller community, and the extent of its contribution to mortality and morbidity rates is relatively limited.

With respect to certain health conditions, general information is available in relation to mortalities in Irish Travellers. The All Ireland Traveller Health Study (AITHS) found that 13\% of General Register Office-confirmed deaths in Irish Travellers in Ireland during a 1-year period (October 2007 to October 2008) were due to respiratory disease. The standardized mortality ratios due to respiratory disease were about 5.4- and 7.5-fold higher in Traveller females and males, respectively, than in the general Irish population [1]. Data outlining the proportion of Irish Traveller deaths in Ireland that were caused by respiratory infectious disease are not currently available. This limits the characterization of differential respiratory disease rates, and their associated determinants.

Tuberculosis (TB) is the leading cause of mortality due to respiratory infection worldwide, killing $\sim 1.5$ million people each year [4]. Using routine Irish enhanced TB surveillance data [5], we examined in this work the available case notification data with respect to TB in the Irish Traveller population.

\section{METHODS}

TB disease notifications for the period from 2002 to 2013 were extracted from the National TB Surveillance System and the Computerised Infectious Disease Reporting (CIDR) system (since 2011) by the Health Protection Surveillance Centre (HPSC). These data are collected by local departments of areas in Ireland on each case of TB notified. Crude incidence rates (CIR) based on reported ethnicity were calculated using denominator data from the Census 2002, 2006, and 2011 data from the Central Statistics Office (CSO), Ireland [6], and also the AITHS data [1] on Traveller population size. Crude incidence rates (CIR)/100000 population were determined for each year from 2002 to 2013. Five-year cumulative CIR values were calculated for the period 2009-2013. Stratification of case based TB notifications by association with an outbreak is available from 2011 onwards. Average prevalence rates for the period 2002-2013 were determined for each age group using Census data from the CSO [6]. Ethical

approval for this study was obtained from the St James's Hospital/Adelaide \& Meath National Children's Hospital Research Ethics Committee, Dublin, Ireland (reference: 2014/037/2014/02).

\section{RESULTS}

As illustrated in Figure 1, the CIR of TB in the Irish Traveller population, based on the number of cases notified, was at a level of $<5$ cases/100000 population per annum from 2002 to 2009. From 2010 onwards, an increase in notifications of TB cases in Irish Travellers was recorded. This resulted in a CIR $>10$ cases/100000 population per annum in 2011 to 2013 using the CSO Traveller population enumeration. In the general population during the same period, the CIR decreased from $10 \cdot 5$ new TB cases/100 000 population [95\% confidence interval (CI) $9 \cdot 5-11 \cdot 5]$ in 2002 to $8 \cdot 3 / 100000$ population $(95 \%$ CI $7 \cdot 5-9 \cdot 1)$ in 2013 . The CIR for TB in Irish Travellers was about threefold higher than that of the white Irish-born population in the years 2011 and 2012. In 2013, an outbreak of TB in the Irish Traveller population resulted in a CIR of $40 \cdot 6 / 100000(95 \%$ CI $21 \cdot 0$ $71 \cdot 1)$ that was about tenfold higher than the corresponding rate in the white Irish-born population. When excluding outbreak cases, the CIR of TB in the Traveller population for 2013 was still higher $(16 \cdot 9 / 100000,95 \%$ CI $5 \cdot 5-39 \cdot 6)$ than that of the general population $(8 \cdot 3 / 100000,95 \%$ CI $7 \cdot 5-9 \cdot 1)$, and of the white Irish-born population $(4 \cdot 2 / 100000,95 \%$ CI 3.5-4.8) (Fig. 1).

To allow for annual variations in TB cases in different populations, 5-year cumulative crude incidence rate values were calculated for the period 20092013. As illustrated in Table 1, the 5 -year cumulative CIR of TB in Irish Travellers was 81.4/100000 (95\% CI 29.6-197.7) compared to $45 \cdot 5 / 100000(95 \%$ CI $41 \cdot 9-50 \cdot 8)$ in the general population and $27 \cdot 3 / 100000$ $(95 \%$ CI $22 \cdot 9-30 \cdot 3)$ in the white Irish-born population for 2009-2013.

The CIR is dependent upon the population data used for Irish Travellers. The total number of Irish Travellers in Ireland, enumerated from the April 2011 Census by the CSO, was $29573(0 \cdot 64 \%)$ of the total population [6]. The total number of Irish Travellers in Northern Ireland, enumerated from the March 2011 Census by the Northern Ireland Statistics and Research Agency, was 1301 (0.07\%) of the total population [7]. This corresponds to an Irish Traveller population size in the region of 30874 for 


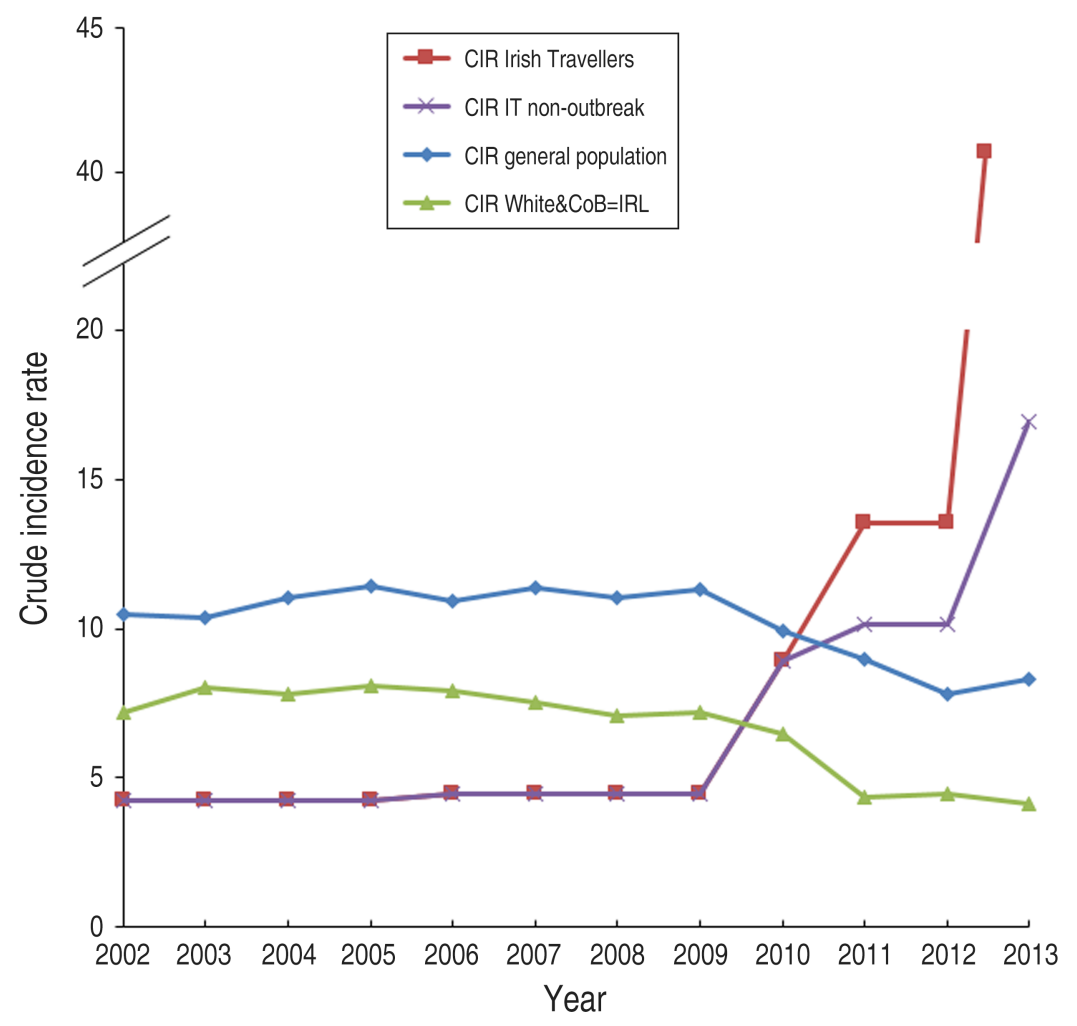

Fig. 1. Crude incidence rate (CIR)/100000 of TB in Ireland from 2002 to 2013. CIR of TB in the general population, white Irish-born in Ireland (white\&CoB $=\mathrm{IRL}$ ) and Irish Travellers are illustrated. The CIR in Irish Travellers excluding outbreak cases (IT non-outbreak) is also illustrated.

Table 1. Cumulative notifications and crude incidence rates of TB from 2009 to 2013*

\begin{tabular}{llll}
\hline \hline & $\begin{array}{l}\text { Cumulative no. of TB } \\
\text { notifications, 2009-2013 }\end{array}$ & $\begin{array}{l}\text { Cumulative TB CIR, } \\
\text { 2009-2013 (Census 2011 } \\
\text { population) }\end{array}$ & $\begin{array}{l}\text { Cumulative TB CIR, } \\
\text { 2009-2013 (AITHS } \\
\text { population) }\end{array}$ \\
\hline $\begin{array}{l}\text { All ethnicity } \\
\text { (including unknown) }\end{array}$ & 2060 & $45 \cdot 5$ & n.a. \\
$\begin{array}{l}\text { White \& CoB = IRL } \\
\begin{array}{l}\text { Irish Traveller } \\
\text { CoB = IRL + UK }\end{array}\end{array}$ & 983 & $27 \cdot 3$ & n.a. \\
\hline \hline
\end{tabular}

CIR, Crude incidence rate/100000; AITHS, All Ireland Traveller Health Study; CoB, country of birth; n.a., not available.

* Notification data for 2013 are provisional.

the island of Ireland in March/April 2011. By contrast, the AITHS conducted from 2007 to 2010 estimated the Irish Traveller population to be 36224 in Ireland and 3905 in Northern Ireland, or 40129 for the island of Ireland. As can be seen from Table 1, where the AITHS population data is used instead of the official Census data, the CIR of TB in the Irish Traveller population is lower. Where the higher enumeration of the Traveller population size from the AITHS is used, the 5-year cumulative CIR was still higher in Irish Travellers than in the general population and in the white Irish-born population (Table 1).

When the average prevalence of TB for the period 2002-2013 is analysed by age, notable differences emerge between Irish Traveller cases and those in the general and white Irish-born populations. Figure 2 shows age-specific rates by Irish Traveller ethnicity from 2002 to 2013 . The majority of cases in Irish Travellers were in the $0-4$ to $25-34$ years age groups with a mean age of 26 years and median 


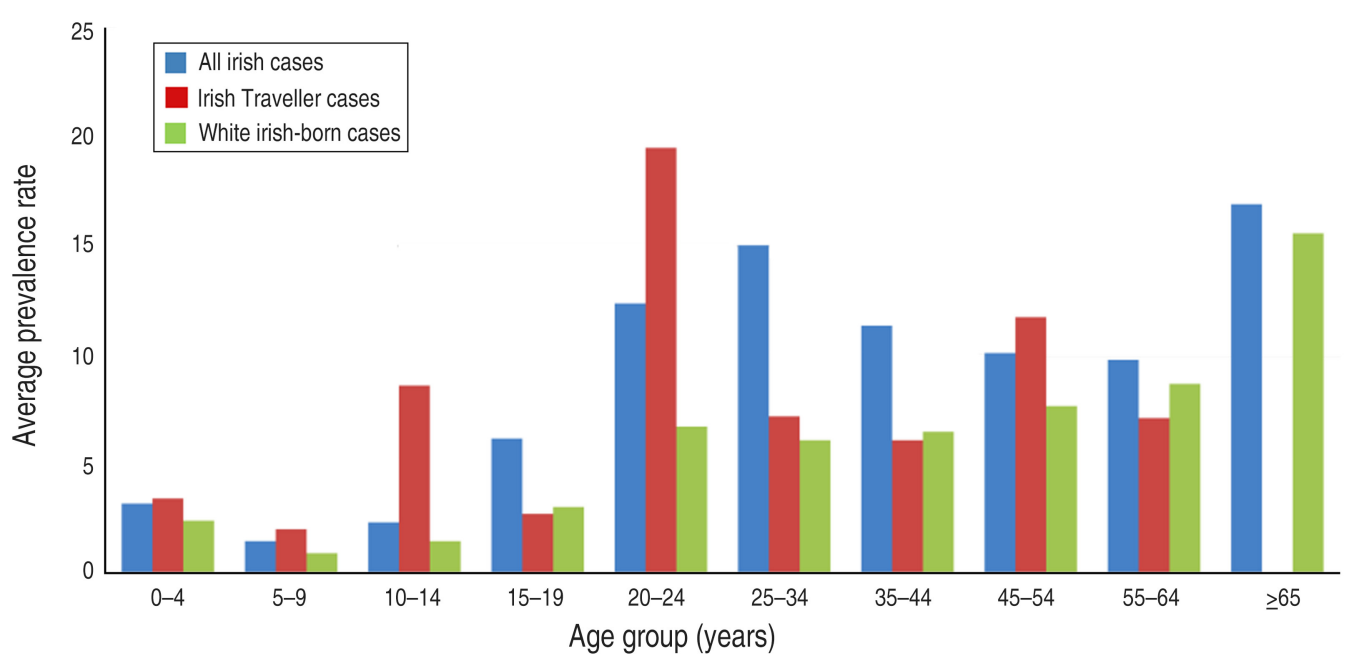

Fig. 2. Average TB prevalence rate (per 100000) for the period 2002-2013 by age group (years) and age-specific rates by Irish Traveller ethnicity, and white Irish-born ethnicity.

age of 24 years. In the general population, the majority of cases occurred in the 25-34 to $\geqslant 65$ years age groups with a mean age of 43 years and median age of 38 years. In the white Irish-born population, the majority of cases occurred in the 55-64 and $\geqslant 65$ years age groups with a mean age of 49 years and a median age of 49 years.

\section{DISCUSSION}

As recently noted by Tollefson et al:: "the paucity of published information on TB burden among indigenous peoples highlights the need to implement and improve TB surveillance to better measure and understand global disparities in TB rates' [8]. For indigenous minority populations in the USA, the ethnicity data available have revealed that American Indians and Alaskan Natives have a 5·4-fold higher rate of $\mathrm{TB}$ compared to non-Hispanic Caucasians. The TB incidence rate in the Aboriginal population is 5.3- and 12.9-fold higher in Australia and Canada, respectively, compared to the domestic-born non-Aboriginal population [9, 10]. In New Zealand, Māori have an approximate sixfold higher rate of TB compared to people of European descent $[11,12]$. An earlier study conducted on Roma in Barcelona found that they have a TB incidence rate which is $5 \cdot 3$-fold higher than the national incidence rate for Spain [8]. While one cannot deduce from the above findings alone that Irish Travellers as an indigenous minority experience a higher rate of TB with respect to the general population, they share with indigenous minority populations in other countries a number of determinants of poor health status such as lower levels of employment and education attainment, as well as social exclusion. While studies in the UK have identified higher numbers of Gypsy Travellers (including Irish, English, Scottish and Welsh Gypsies and Travellers) reporting illnesses that include bronchitis and asthma with respect to an age-matched comparator group [13, 14], data with respect to the incidence of TB in Irish Travellers in the UK have not been reported [15].

As a first step in examining the incidence of TB in the Irish Traveller population, we examined TB case notifications in Ireland for the period from 2002 to 2013. Based on the available data, notified cases of TB in Irish Travellers increased from $4 \cdot 2$ cases $/ 100$ 000 in 2002 to more than $10 / 100000$ from 2011 onwards. In comparison, notified cases of TB in the general population decreased from $10 \cdot 5$ cases $/ 100$ 000 in 2002 to $8 \cdot 3 / 100000$ in 2013. The available notification data from 2011 onwards indicate a more than threefold higher incidence rate of TB in Irish Travellers compared to their white Irish-born compatriots (Fig. 1). This differential is also seen in the respective 5-year CIR of TB for the period 2009-2013 for Irish Travellers $(81 \cdot 4 / 100000)$ and the white Irish-born population $(27 \cdot 3 / 100000)$ (Table 1). In terms of the age-specific distribution of TB cases, the mean age of cases in Irish Travellers (26 years) was notably lower than that of the general population (mean age of 43 years) and of the white Irish-born population (mean age of 49 years).

Studies in different jurisdictions may provide an insight into why the incidence of TB could be higher in 
Irish Travellers. Socioeconomic position is known to affect people's health. As noted by Fiscella \& Williams: 'Differences in socioeconomic status, whether measured by income, educational achievement, or occupation, are associated with large disparities in health status. This association persists across the life cycle and across measures of health, including health status, morbidity, and mortality. Although effects are largest for those living in poverty, gradients of disparity are seen across the socioeconomic spectrum' [16]. More recently, Boccia and colleagues established a specific association between household socioeconomic position and TB [17]. It is widely accepted that Irish Travellers experience a lower socioeconomic status as indicated by parameters such as unemployment and education. Unemployment was measured at $84 \cdot 3 \%$ in the Irish Traveller community (percentage of people in the labour force who were either looking for their first job or unemployed) in the Irish Census 2011 [6]. This compared to $14 \cdot 6 \%$ unemployment in the general population in 2011. In terms of education, only $3 \cdot 1 \%$ of Irish Travellers continued their education beyond the age of 18 years in contrast to $41 \cdot 2 \%$ of the general population [6].

A lower socioeconomic status in Irish Travellers may coincide with a higher prevalence of one or more known risk factors for the development of TB. Poor accommodation has previously been cited as a factor in the lower health status of Irish Travellers [1]. According to the Irish Census 2011, the average number of rooms in Irish Traveller households was 4.3 compared to $5 \cdot 5$ rooms for all private households in the state in 2011. Two and a half per cent of Irish Traveller households have $\geqslant 10$ persons compared to $0.04 \%$ of non-Irish Traveller households. Therefore, Irish Traveller households have a higher occupant density compared to non-Traveller households. It is worthy of note that in New Zealand, household crowding has been identified as a risk factor for increased TB incidence [18], and that exposure to extreme crowding was high in the indigenous Māori population compared to the European/Other group [19]. According to the Irish Census 2011, 'between 2006 and 2011 the percentage of Irish Traveller households residing in caravans or mobile/temporary structures halved from $24.7 \%$ to $12.3 \%$ ' [6]. Whether a transition from traditional nomadism to so-called 'settled' accommodation has impacted on risk factors for TB in Irish Travellers would require in-depth investigation.

In terms of other known risk factors for the development of TB, a study published in 2009 reported that the prevalence of pre-diabetes and diabetes in the Irish Traveller population was about twofold higher than in the background population in Ireland [20]. In the UK, relatively high rates of smoking prevalence have been reported for Irish Traveller males and females [14, 21]. Further research is needed to establish and quantify each of the specific risk factors that underlie a higher incidence of TB in Irish Travellers. This information would be valuable in guiding intervention measures to reduce the risk of TB development in Irish Travellers.

Although this study illustrates differences in the incidence of TB in the Irish Traveller population compared to the general and white Irish populations, a number of limitations of the study should be noted. One limitation pertains to the completeness of the available ethnicity data. The proportion of TB cases in the Irish Traveller population that was correctly identified with respect to ethnicity is not known. A similar limitation was reported recently with respect to an assessment of meningococcal surveillance data in Ireland [22]. The definition of the Irish Traveller community in Irish Law (Equal Status Act, 2000, Part I) is given as follows: "Traveller community" means the community of people who are commonly called Travellers and who are identified (both by themselves and others) as people with a shared history, culture and traditions including, historically, a nomadic way of life on the island of Ireland.' In the Irish Census 2011, people were asked the question: 'What is your ethnic or cultural background?' Hence, for census purposes, self-declaration of ethnicity is used. In terms of the surveillance of notifiable infectious diseases, it is possible for local departments of public health to record ethnicity for all notifications of notifiable diseases as per the CSO ethnicity categories. However, it is not clear if the practice of selfdeclaration of Irish Traveller ethnicity is in widespread use with respect to notifiable disease surveillance in Ireland. Incomplete recording of ethnicity could potentially result in an underestimation of the number of TB cases in the Traveller community. National guidance on the standardized collection of ethnicity identifiers has been noted as a possible future project within the HPSC of the HSE, Ireland in conjunction with relevant stakeholders.

Another limitation of the study relates to the relatively small population size of Irish Travellers who constitute $0.64 \%$ of the total population in Ireland [6]. An outbreak of TB in the Traveller population can significantly affect the CIR/100000. This is evident 
in wide confidence intervals that were obtained with respect to CIRs for Irish Traveller cases. In 2013, an outbreak of TB in the Irish Traveller community resulted in a CIR of $40 \cdot 6 / 100000$ per annum, using the CSO traveller population as a denominator. Due to the impact of this outbreak on the incidence rate, we examined the data excluding cases linked to the outbreak. The incidence rate of $\mathrm{TB}$ in Irish Travellers in 2013 was still higher (16.9/100000) than in the general population $(8 \cdot 3 / 100000)$ and in the white Irish-born population $(4 \cdot 2 / 100000)$ when Irish Traveller outbreak TB cases were excluded (Fig. 1).

With an increase in notifications of a notifiable disease, there is often the consideration of whether it may be due to enhanced surveillance, or due to a true increase in incidence. An earlier study found that undernotification of TB in different studies in the UK ranged from $7 \%$ to $27 \%$ of cases [23]. For the whole of the UK, data completeness with respect to known ethnicity of TB cases was 98\% in 2013 [15]. The completeness of the ethnicity data for TB cases in Ireland before an increase in $\mathrm{TB}$ notifications in Irish Travellers stood at $97.9 \%$ in 2009 , compared to ethnicity data completeness of $83.5 \%$ in 2013 where notifications in Irish Travellers were highest. Therefore, a surge in ethnicity reporting for all TB cases in Ireland would not appear to be responsible for the increase in TB cases in Irish Travellers. This does not preclude the possibility of a differential enhancement of TB surveillance in a given population group such as Irish Travellers. At this stage, it is not possible to conclude whether enhanced surveillance or increased incidence, or both, are responsible for the increase in TB notifications in Irish Travellers. Nevertheless, the data point to a dissimilar incidence rate and age distribution of TB in Irish Travellers with respect to the general and white Irish populations and provide evidence that the monitoring of TB over a longer period in Irish Travellers is warranted.

In terms of ethnicity identifier use for Irish Travellers, the Irish Department of Health and Children's document 'Traveller Health: A National Strategy 2002-2005' made the following points:

Making significant progress in tackling Traveller health status will be difficult unless an adequate system can be put in place to gather data on an ongoing basis on Traveller health. This data, effectively the baseline from which progress can be measured and by which services can be planned and monitored, is now an urgent necessity. However, for the purpose of effectively gathering information on the health status of the Traveller community, it may be necessary to modify existing health information systems in order to identify Travellers as an ethnic group [24].

The above recommendation has been employed by HPSC with regard to the use of enhanced surveillance forms for $\mathrm{TB}$, invasive meningococcal disease/bacterial meningitis, salmonellosis, which specifically identify Irish Travellers under ethnicity. In addition, the CIDR system, which is used to record all cases of notifiable infectious disease in Ireland, contains a core variable which allows ethnicity to be recorded for every case of notifiable disease reported. These developments, where applied routinely with case investigation, would reduce passive reporting or thirdparty declaration of Irish Traveller ethnicity in disease surveillance, and consequently, increase the completeness and robustness of Irish Traveller health data.

In summary, it has been reported previously that the population age structure of the Irish Traveller population in Ireland resembles that of a developing country with high mortality rates at a younger age [1]. The extent to which infectious diseases may contribute to this observation deserves investigation. Acknowledging the limitations of the data available, this study constitutes one of the first reports to examine the incidence of the respiratory disease, TB, in the Irish Traveller population. We detected in our study a higher incidence rate of TB in Irish Travellers relative to the general population, and in particular, with respect to the white Irish-born population. With the general decline in TB in the white Irish-born population, TB cases may be concentrated in marginalized groups such as Irish Travellers. Routine recording of Irish Traveller ethnicity for TB, as well as other notifiable infectious diseases, could strengthen the detection of inequities in infection rates and the characterization of their respective determinants. This would in turn provide guidance on appropriate preventive measures that may reduce communicable disease morbidity and mortality in the Irish Traveller population.

\section{ACKNOWLEDGEMENTS}

The authors thank Ronnie Fay and Nurul Amin at the Pavee Point Traveller and Roma Centre, Dublin, for their valued discussions.

\section{DECLARATION OF INTEREST}

None. 


\section{REFERENCES}

1. All Ireland Traveller Health Study Team. All Ireland Traveller Health Study, 2010.

2. Office for National Statistics. 2011 Census for England and Wales.

3. Baker M. Gypsies and Travellers-Leeds Baseline Census 2004-2005. 2005.

4. World Health Organisation. Global tuberculosis report, 2014.

5. Health Protection Surveillance Centre. National TB surveillance, a report by the Health Protection Surveillance Centre, Quarter 1-4, 2013 TB Report, 2014.

6. Central Statistics Office. Census of Ireland 2011.

7. Northern Ireland Statistics and Research Agency. Census 2011.

8. Tollefson D, et al. Burden of tuberculosis in indigenous peoples globally: a systematic review. International Journal of Tuberculosis and Lung Disease 2013; 17: 1139-1150.

9. Barry C, et al. Tuberculosis notifications in Australia, 2008 and 2009. Communicable Diseases Intelligence Quarterly Report 2012; 36: 82-94.

10. Public Health Agency of Canada. Tuberculosis in Canada 2008. 2012.

11. Bissielo A, Lim E, Heffernan H. Tuberculosis in New Zealand: Annual Report 2011. Institute of Environmental Science and Research Ltd.

12. Lim E, Heffernan H. Tuberculosis in New Zealand: Annual Report 2012. Institute of Environmental Science and Research Ltd.

13. Parry G, et al. The health status of gypsies and travellers in England. University of Sheffield, 2004.

14. Parry G, et al. Health status of Gypsies and Travellers in England. Journal of Epidemiology and Community Health 2007; 61: 198-204.
15. Public Health England. Tuberculosis in the UK - 2014 report. 2014.

16. Fiscella K, Williams DR. Health disparities based on socioeconomic inequities: implications for urban health care. Academic Medicine 2004; 79: 1139-1147.

17. Boccia D, et al. The measurement of household socioeconomic position in tuberculosis prevalence surveys: a sensitivity analysis. International Journal of Tuberculosis and Lung Disease 2013; 17: 39-45.

18. Baker M, et al. Tuberculosis associated with household crowding in a developed country. Journal of Epidemiology and Community Health 2008; 62: 715-721.

19. Baker M, et al. Infectious diseases attributable to household crowding in New Zealand: a systematic review and burden of disease estimate. In He Kainga Orangal Housing and Health Research Programme, University of Otago, 2013.

20. Tan S, et al. Traveller health: prevalence of diabetes, pre diabetes and the metabolic syndrome. Irish Medical Journal 2009; 102: 176-178.

21. Aspinall PJ, Mitton L. Smoking prevalence and the changing risk profiles in the UK ethnic and migrant minority populations: implications for stop smoking services. Public Health 2014; 128: 297-306.

22. Cotter S, et al. Meningococcal disease in Ireland - can determinants of increased risk be identified? Epi-Insight 2014; 15: 1-5.

23. Pillaye J, Clarke A. An evaluation of completeness of tuberculosis notification in the United Kingdom. BMC Public Health 2003; 3: 31.

24. Department of Health and Children. Traveller health: a national strategy 2002-2005. 2002. 